\title{
DHFR and MDR1 upregulation is associated with chemoresistance in osteosarcoma stem-like cells
}

\author{
YU-HSIEN LEE ${ }^{1,2^{*}}$, HUI-WEN YANG ${ }^{1,2^{*}}$, LI-CHIU YANG ${ }^{1,2}$, MING-YI LU $^{1,2}$, LO-LIN TSAI $^{1,2}$, SHUN-FA YANG $^{3}$, \\ YU-FENG HUANG ${ }^{1,2}$, MING-YUNG CHOU ${ }^{1,2,4}$, CHENG-CHIA YU ${ }^{1,2,4}$ and FANG-WEI HU ${ }^{1,2}$ \\ ${ }^{1}$ School of Dentistry, Chung Shan Medical University; ${ }^{2}$ Department of Dentistry, Chung Shan Medical University Hospital; \\ ${ }^{3}$ Institute of Medicine; ${ }^{4}$ Institute of Oral Sciences, Chung Shan Medical University, Taichung 40201, Taiwan, R.O.C.
}

Received March 17, 2015; Accepted July 1, 2016

DOI: $10.3892 / \mathrm{ol} .2017 .6132$

\begin{abstract}
Tumor-initiating cells (TICs) are defined as a specialized subset of cells with tumor-initiating capacity that can initiate tumor growth, tumor relapse and metastasis. In the present study, osteosarcoma TICs (OS-TICs) were isolated and enriched from the osteosarcoma U2OS and MG-63 cell lines using sphere formation assays and serum-depleted media. These enriched OS-TICs showed the expression of several typical cancer stemness markers, including octamer-binding transcription factor 4, Nanog homeobox, cluster of differentiation (CD)117, Nestin and CD133, and the expression of ATP binding cassette subfamily $\mathrm{G}$ member 2 , multidrug resistance protein 1 (MDR1) and dihydrofolate reductase (DHFR). Notably, in vitro and in vivo tumorigenic properties were enhanced in these OS-TICs. Additionally, methotrexate and doxorubicin are the most widely used anticancer agents against osteosarcoma, and the observed enhanced chemoresistance of OS-TICs to these two agents could be associated with the upregulation of DHFR and MDR1. These findings suggest that the upregulation of DHFR and MDR1 is associated with the development of chemoresistance of OS-TICs.
\end{abstract}

\section{Introduction}

Osteosarcoma, a highly malignant and aggressive tumor generated from mesenchymal tissue that produces osteoid and bone, occurs more frequently in the early years of life (1).

Correspondence to: Dr Cheng-Chia Yu, Institute of Oral Sciences, Chung Shan Medical University, 110 Sec. 1 Jianguo N. Road, Taichung 40201, Taiwan, R.O.C.

E-mail: ccyu@csmu.edu.tw

Dr Fang-Wei Hu, School of Dentistry, Chung Shan Medical University, 110 Sec. 1 Jianguo N. Road, Taichung 40201, Taiwan, R.O.C.

E-mail: fang0989909009@gmail.com

*Contributed equally

Key words: tumor-initiating cells, chemo-resistance, multidrug resistance protein 1 , dihydrofolate reductase
Osteosarcoma develops rapidly and is usually associated with high mortality due to its progressive pulmonary metastasis, which eventually leads to respiratory failure (1). Despite the development of the multidisciplinary treatment for osteosarcoma, the prognosis of osteosarcoma patients remains poor (1).

Tumor-initiating cells (TICs), also known as cancer stem cells (CSCs), comprise a unique subpopulation of cells within a tumor. Relative to the remainder of the tumor bulk, CSCs are often more tumorigenic and chemoresistant or radioresistant in comparison (2). Evidence has indicated that novel therapeutics targeting CSCs, which are critical for tumorigenicity and tumor progression, could significantly improve the clinical outcome of cancer treatment (2). Therefore, it is of paramount importance to uncover the existence of CSCs and to elucidate the pathogenesis of osteosarcoma.

Octamer-binding transcription factor 4 (Oct-4), a member of the POU-domain transcription factors family, is a key reprogramming factor that balances the pluripotent and differentiated states in stem cell and cancer development (3). Notably, Oct-4 is crucial role for regulating the self-renewal of stem cells, and its expression dowregulates substantially during cell differentiation (4). Nanog homeobox (Nanog), another transcription regulator, is a key factor for maintaining pluripotency during embryonic development (5). The messenger RNA (mRNA) expression of Nanog is abundant in pluripotent cell lines, including embryonic stem, embryonic germ and embryonic carcinoma cells, compared with in adult tissues (5). Similar to Oct-4, Nanog expression is also downregulated when pluripotent cells undergo differentiation (5). Collectively, these findings indicate the possibility that Oct-4 and Nanog may also be important in stem-like cells derived from osteosarcoma.

Dihydrofolate reductase (DHFR), an enzyme that reduces dihydrofolic acid to tetrahydrofolic acid, is important for the synthesis of nucleic acid precursors, which are used for cell proliferation and growth (6). DHFR, the target of methotrexate (MTX) in osteosarcoma chemotherapy (6), is demonstrably important for the development of chemoresistance to MTX in human osteosarcoma cells $(7,8)$. The reemergence of chemotherapy-resistant CSCs has been shown to contribute to cancer relapse following conventional therapeutic treatment (9). However, the functional role of DHFR in chemoresistance in CSCs has not been determined. 
To further elucidate the roles of Oct- 4 and Nanog in the tumorigenesis of osteosarcoma, the present study aimed to compare and distinguish the phenotypic differences between osteosarcoma TICs (OS-TICs) and parental osteosarcoma cells, as well as isolate the OS-TICs using sphere formation assays and defined serum-depleted media containing basic fibroblast growth factor (bFGF) and epidermal growth factor (EGF). In addition, the present study sought to evaluate the expression of several stem-like markers, including Oct-4, Nestin, Nanog, cluster of differenatiation (CD)117 and CD133, and the anticancer drug transporters, MDR1 and ATP binding cassette subfamily G member 2 (ABCG2). The capabilities of OS-TICs for migration, invasion and tumorigenicity were simultaneously assessed in vitro and in vivo. The findings of the present study may help to elucidate the mechanisms underlying the chemoresistance in osteosarcoma patients.

\section{Materials and methods}

Isolation of OS-TICs from osteosarcoma cells by tumor sphere-forming assay. The human osteosarcoma cell lines (U2OS and MG-63) were purchased from the American Type Culture Collection (Manassas, VA, USA). Originally, U2OS and MG-63 cells were grown in Dulbecco's modified Eagle's medium (DMEM) (Gibco; Thermo Fisher Scientific, Inc., Waltham, MA, USA) supplemented with $10 \%$ fetal bovine serum (HyClone; GE Healthcare Life Sciences, Logan, UT, USA). U2OS and MG-63 cells were cultured as sphere-forming OS-TICs, as described previously (10). For expanding OS-TICs, primary tumor spheres were dissociated into single cell suspension using HyQTase solution (HyClone; GE Healthcare Life Sciences) at $37^{\circ} \mathrm{C}$ for $5 \mathrm{~min}$, and seeded at a density of $10^{4}$ live cells $/ 10-\mathrm{mm}$ into ultralow attachment 6-well plates (Corning Life Sciences, Tewksbury, MA, USA), with defined serum-depleted DMEM/ F-12 (Gibco; Thermo Fisher Scientific, Inc.) containing $10 \mathrm{ng} /$ $\mathrm{ml} \mathrm{bFGF} \mathrm{(Invitrogen;} \mathrm{Thermo} \mathrm{Fisher} \mathrm{Scientific,} \mathrm{Inc.)} \mathrm{and} 10 \mathrm{ng} /$ ml EGF (Invitrogen; Thermo Fisher Scientific, Inc.).

RNA extraction, RNA quantification and quantitative reverse transcription-polymerase chain reaction ( $q R T-P C R)$. Total RNA was extracted from cells using RNeasy Mini Kits (Qiagen, Inc., Valencia, CA, USA), according to the manufacturer's instructions. RNA concentration was measured using a NanoVue Plus spectrophotometer (GE Healthcare Life Sciences). RNA was then reverse transcribed to complementary DNA using the SuperScript III First-Strand Synthesis System (Invitrogen; Thermo Fisher Scientific, Inc.). The TaqMan mRNA Assay (Applied Biosystems; Thermo Fisher Scientific, Inc.) was used to determine the mRNA expression levels on the StepOnePlus Fast Real-Time PCR system (Applied Biosystems; Thermo Fisher Scientific, Inc.). The cycling conditions were as follows: $50^{\circ} \mathrm{C}$ for $2 \mathrm{~min}, 95^{\circ} \mathrm{C}$ for $10 \mathrm{~min}$, and 40 cycles of $95^{\circ} \mathrm{C}$ for $10 \mathrm{sec}$ and $60^{\circ} \mathrm{C}$ for $1 \mathrm{~min}$. Endogenous control gene, GAPDH, was used to normalize the relative gene expression level. Primer sequences listed in Table I were synthesized and purchased from Mission Biotech (Taipei, Taiwan). Foldchanges were determined using the $2^{-\Delta \Delta C q}$ method (11).

Western blot analysis. Parental osteosarcoma or OS-TICs were lysed using NP-40 lysis buffer (Sigma-Aldrich; Merck KGaA,
Darmstadt, Germany), and the protein concentration was determined using bicinchoninic acid protein assay reagent (Thermo Fisher Scientific Inc.). Samples ( $25 \mu \mathrm{g}$ of total protein) were boiled at $95^{\circ} \mathrm{C}$ for $5 \mathrm{~min}$ and separated by $10 \%$ SDS-PAGE. The proteins were wet-transferred to polyvinylidene difluoride membranes (EMD Millipore, Billerica, MA, USA). After blocking with $5 \%$ milk for $30 \mathrm{~min}$, the membranes were washed three times with $0.1 \%$ Tween-20 in Tris buffer (TBS-T) for $5 \mathrm{~min}$ each. Subsequently, the primary antibodies were diluted in $0.1 \%$ TBS-T and incubated with the membrane at room temperature for $1 \mathrm{~h}$. Upon washing three times for $5 \mathrm{~min}$ each with $0.1 \%$ TBS-T, the membrane was incubated for $30 \mathrm{~min}$ at room temperature with horseradish peroxidase-conjugated anti-rabbit (1:3,000; catalog no. 7074; Cell Signaling Technology, Inc., Danvers, MA, USA) or anti-mouse immunoglobulin $\mathrm{G}$ polyclonal antibodies (1:3,000; catalog no. 7076; Cell Signaling Technology, Inc.) were used as secondary antibodies diluted with $0.1 \%$ TBS-T. Following three washes with $0.1 \%$ TBS-T for $5 \mathrm{~min}$ each, the signals were developed using an Enhanced Chemiluminescence Plus substrate (PerkinElmer, Inc., Waltham, MA, USA) and captured using a LAS-1000plus Luminescent Image Analyzer (GE Healthcare Life Sciences). The following primary antibodies were used: Anti-Oct-4 antibody (1:1,000; \#2750; Cell Signaling Technology, Inc.), anti-Nanog antibody (1:500; \#3850; Cell Signaling Technology, Inc.), anti-Nestin antibody (1:1,000; MAB5326; EMD Millipore), anti-GAPDH antibody (1:2,000; GTX627408; GeneTex, Inc., Irvine, CA, USA), anti-DHFR antibody (1:1,000; ab49881; Abcam, Cambridge, UK) and anti-MDR1 antibody (1:1,000; \#13978; Cell Signaling Technology, Inc.).

Immunofluorescent staining. Parental and OS-TICs cells were plated on glass coverslips and then fixed with $4 \%$ buffered formalin in PBS. Cells were permeabilized with $0.1 \%$ Triton X-100/PBS, washed with PBS, and incubated with a mouse anti-Nestin monoclonal antibody (1:100; MAB5326; EMD Millipore), an antibody against Oct-4 (1:50; \#2750; Cell Signaling Technology, Inc.), a rabbit polyclonal antibody against Nanog (1:50; \#3850; Cell Signaling Technology, Inc.) or a rabbit anti-MDR1 monoclonal antibody (1:100; \#13342; Cell Signaling Technology, Inc.). Upon washing with $0.1 \%$ Tween-20 in PBS, the cells were then incubated with fluorescent probe-conjugated secondary antibodies $(1: 1,000$; \#Z25002; Molecular Probes; Thermo Fisher Scientific, Inc.) at room temperature for $1 \mathrm{~h}$. Cells were further counterstained with $0.1 \mu \mathrm{g} / \mathrm{ml}$ DAPI. The images were captured using the Leica Application Suite X with Leica DMi8 (Leica Microsystems $\mathrm{GmbH}$, Wetzlar, Germany).

Flow cytometry analysis. Parental and OS-TICs cells were stained with fluorescein isothiocyanate-labeled anti-CD133 (1:100; 130-105-225; Miltenyi Biotech, Inc., Auburn, CA, USA), anti-CD117 (1:100; 313232; BioLegend, Inc., San Diego, CA, USA) or anti-ABCG2 (1:10; 130-104-957; Miltenyi Biotech, Inc.) antibodies, according to the manufacturer's instructions. All antibodies were diluted in $0.1 \%$ bovine serum albumin (A8531; Sigma-Aldrich; Merck KGaA). The results were measured using the FACSCalibur flow cytometer (BD Biosciences, Franklin Lakes, NJ, USA) and analyzed by FlowJo 7.6 software (FlowJo, LLC, Ashland, OR, USA). 
Table I. The sequences of the primers for quantitative reverse transcription-quantitiative polymerase chain reaction.

\begin{tabular}{|c|c|c|c|}
\hline Gene (accession no.) & Primer sequence, $5^{\prime}$ to $3^{\prime}$ & Product size, base pairs & Temperature, ${ }^{\circ} \mathrm{C}$ \\
\hline $\begin{array}{l}\text { Oct-4 } \\
(\text { NM_002701) }\end{array}$ & $\begin{array}{l}\text { F: GTGGAGAGCAACTCCGATG } \\
\text { R: TGCTCCAGCTTCTCCTTCTC }\end{array}$ & 86 & 60 \\
\hline $\begin{array}{l}\text { Nanog } \\
(\text { NM_024865) }\end{array}$ & $\begin{array}{l}\text { F: ATTCAGGACAGCCCTGATTCTTC } \\
\text { R: TTTTTGCGACACTCTTCTCTGC }\end{array}$ & 76 & 60 \\
\hline $\begin{array}{l}\text { GAPDH } \\
\text { (NM_002046) }\end{array}$ & $\begin{array}{l}\text { F: CATCATCCCTGCCTCTACTG } \\
\text { R: GCCTGCTTCACCACCTTC }\end{array}$ & 180 & 60 \\
\hline
\end{tabular}

Oct-4, octamer-binding transcription factor 4; Nanog, Nanog homeobox; F, forward; R, reverse.

Table II. In vivo tumorigenicity of parental U2OS and derived OS-TICs was examined in NOD/SCID mice by xenotransplantation analysis.

\begin{tabular}{lcccc}
\hline & \multicolumn{4}{c}{ No. of cells for injection } \\
\cline { 2 - 5 } Cells & $1 \times 10^{4}$ & $5 \times 10^{4}$ & $1 \times 10^{5}$ & $2 \times 10^{5}$ \\
\hline U2OS & $0 / 3$ & $0 / 3$ & $1 / 3$ & $2 / 3$ \\
OS-TICs & $3 / 3$ & $3 / 3$ & $3 / 3$ & $3 / 3$ \\
\hline
\end{tabular}

OS-TICs, osteosarcoma tumor-initiating cells. Three mice were used per injection group.

Transwell chamber assay for migration/invasion analysis. Cell migration and invasion assays were performed using $5 \times 10^{4}$ parental osteosarcoma or derived OS-TICs in 24-well plate Transwell chambers (pore size, $8 \mu \mathrm{m}$; Millicell; EMD Millipore), as described previously (10). Cell suspensions were seeded into the upper compartment of the Transwell chamber at a cell density of $1 \times 10^{5}$ cells per $100 \mu \mathrm{l}$ in serum-free medium. The lower chamber was filled with medium containing $10 \%$ serum. After $24 \mathrm{~h}$ of incubation, the medium was removed, and the filter membrane was fixed with methanol for $1 \mathrm{~h}$. Subsequently, the remaining cells in the filter membrane facing the lower chamber were stained with crystal violet (Sigma-Aldrich; Merck KGaA). The migrated and invasive cancer cells were then visualized and counted from five different visual fields at x100 magnification under an inverted microscope.

Xenografts animal experiment. All the NOD/SCID mice (18-22 g; National Laboratory Animal Center,Zhunan, Taiwan) practices in the present study were approved and performed in accordance with the Institutional Animal Care and Use Committee of Chung Shan Medical University (Taichung, Taiwan). All mice were housed with a regular 12-h light/dark cycle, and ad libitum access to water and a standard rodent chow diet (Laboratory Rodent Diet 5001; LabDiet, St. Louis, MO, USA), and were kept in a pathogen-free environment at the Laboratory Animal Unit of Chung Shan Medical University (temperature, $22^{\circ} \mathrm{C}$; humidity, 30-70\%; $\mathrm{n}=5$ mice/cage), according to the requirements of the Institutional Animal Care and Use Committee of Chung Shan Medical University, Taichung, Taiwan. Single-cell suspensions containing serial dilutions of parental and OS-TICs in $100 \mu \mathrm{l}$ serum-free medium (Table II) were mixed with $100 \mu$ l Matrigel (BD Biosciences) and subcutaneously injected into 6-week-old, male NOD/SCID mice. Each cell injected group consisted of 3 mice, all of which were male. A total of 24 mice are used for the experiment. At 6 weeks after injection, the mice were sacrificed by $\mathrm{CO}_{2}$ inhalation. Tumor volume (TV) was calculated using the following formula: TV $\left(\mathrm{mm}^{3}\right)=\left(\right.$ length $\mathrm{x}$ width $\left.{ }^{2}\right) / 2$.

MTT assay. The viability of parental and OS-TICs cells treated with increasing concentrations of MTX or doxorubicin was measured by MTT assay (Sigma-Aldrich; Merck KGaA), according to the manufacturer's instructions. Cells were plated in 24-well plates $\left(5 \times 10^{4}\right.$ cells/well $)$ in different concentrations of doxorubicin or MTX and cultured at $37^{\circ} \mathrm{C}$ for $24 \mathrm{~h}$. The concentration of doxorubicin was initiated at $0 \mu \mathrm{M}$ and increased at $50 \mu \mathrm{M}$ increments. The concentration of MTX was also started at $0 \mu \mathrm{M}$, but was increased at $25 \mu \mathrm{M}$ increments. The attached cells were incubated with $0.5 \mathrm{mg} / \mathrm{ml}$ of MTT at $37^{\circ} \mathrm{C}$ for $4 \mathrm{~h}$. The blue formazan crystals of viable cells were dissolved in dimethyl sulfoxide (DMSO) and then evaluated spectrophotometrically at $570 \mathrm{~nm}$. The DMSO-treated group was set as $100 \%$, and data were presented as a percentage of the DMSO control. Cell survival was measured using Infinite M200 PRO (Tecan Group Ltd., Männedorf, Switzerland) and analyzed with Magellan 7.1 software (Tecan Group Ltd.).

Statistical analysis. Data are presented as mean \pm standard error of the mean. Statistical analyses were performed using the unpaired Student's $t$-test in SPSS 16 (SPSS, Inc., Chicago, IL, USA). Kaplan-Meier survival analysis was used to analyze animal survival data. $\mathrm{P}<0.05$ was considered to indicate a statistically significant difference.

\section{Results}

Characterization of progenitor/stem cell properties in isolated OS-TICs. To explore the existence of stem-like cells/TICs in osteosarcoma, the osteosarcoma U2OS and MG-63 cell lines were incubated with defined serum-free medium with bFGF and EGF cultured in low-attachment 6-well plates. After 10 days incubation, the cancer cells gradually detached from the culture dishes, aggregated and became spheres-forming OS-TICs (Fig. 1A). To further characterize the stem-like properties of the enriched OS-TICs, flow cytometry was used to analyze the 


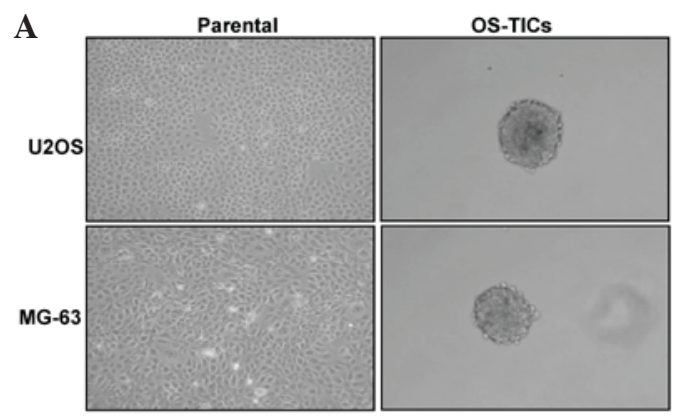

B
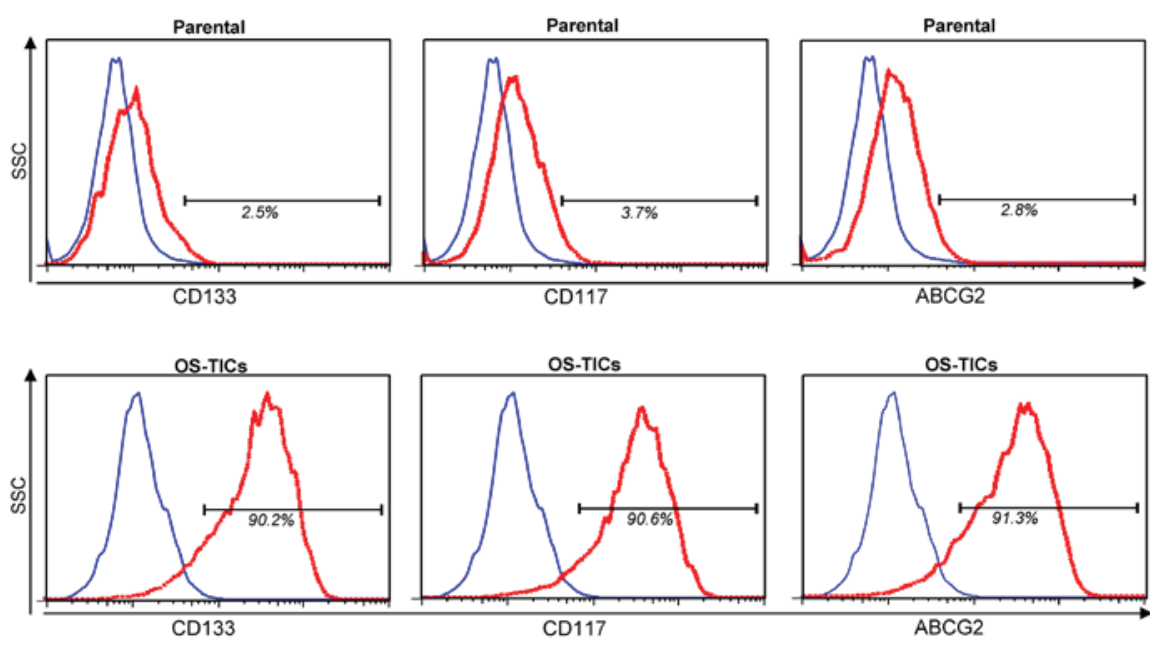

Figure 1. Isolation and characterization of OS-TICs. (A) Osteosarcoma cancer cell lines were used and cultured in serum-depleted Dulbecco's modified Eagle's medium/F-12 medium containing basic fibroblast growth factor and epidermal growth factor into low-attachment 6 -well plates. After 10 days of culture, cancer cells gradually detached from culture dishes, aggregated and formed tumor spheres. (B) Expression profiles of progenitor/stem cell-specific surface markers, including CD133, CD117 and ABCG2, in parental U2OS cells or derived OS-TICs, analyzed by flow cytometry. Single-cell suspension from parental cells or derived OS-TICs was either stained with control immunoglobulin G antibody or experimental antibodies, including anti-CD133 (left), anti-CD117 (middle) and anti-ABCG2 (right). OS-TICs, osteosarcoma tumor-initiating cells; CD, cluster of differentiation; ABCG2, ATP binding cassette subfamily G member 2.

expression profiles of stem-like cell surface markers in parental U2OS cells and OS-TICs. As shown in Fig. 1B, the majority of isolated OS-TICs were positively stained for CD133 and CD117, two well-known cell surface markers of CSCs. Similarly, the upregulation of ABCG2 was also observed to be increased in OS-TICs compared with parental U2OS cells (Fig. 1B). In total, $\sim 90 \%$ of OS-TICs derived from U2OS cells were stained positive for CD133, CD117 and ABCG2.

Elevated expression of progenitor/stem cell genes in OS-TICs. An MTT assay was then used to evaluate the proliferation rate of parental U2OS cells and OS-TICs. Notably, OS-TICs exhibited an increased proliferation capability compared with parental cells (Fig. 2A). The expression of stem cell-specific genes, Oct-4, Nanog, and Nestin, was examined transcriptionally and translationally. The total RNA of parental U2OS cells or OS-TICs was extracted and qRT-PCR was performed. The results confirmed the high expression of Oct-4, Nanog and Nestin transcripts in the enriched OS-TICs (Fig. 2B). Similar to the observations of qRT-PCR, the results of the western blot analysis also showed that the protein levels of Oct-4, Nanog, and Nestin were also upregulated in enriched OS-TICs compared with in parental U2OS cells (Fig. 2C). Furthermore, immunofluorescent staining validated the high levels of Oct-4 (Fig. 2D; top panel), Nestin (Fig. 2D; middle panel), and Nanog
(Fig. 2D; bottom panel) in the intracellular compartments in enriched OS-TICs compared with in parental U2OS cells.

Enhanced tumorigenic potential of OS-TICs in vitro and in vivo. To evaluate whether these enriched OS-TICs possess enhanced tumorigenicity, an in vitro Matrigel assay combined Transwell invasion/migration assay was performed. The results indicated that enriched OS-TICs have increased invasion and migration capabilities compared with the parental U2OS cells (Fig. 3A and B; $\mathrm{P}<0.05$ ). To further validate the enhanced tumor-initiating abilities of OS-TICs in vivo, the parental cells and OS-TICs derived from U2OS cells were injected into the subcutaneous space of NOD/SCID mice for xenograft tumorigenicity assay. As shown in Table II, only one recipient of U2OS parental cells at $1 \times 10^{5}$ cells/mice resulted in a tumor. However, all of three recipients of U2OS OS-TICs developed tumor-like formation when only $1 \times 10^{4}$ cells were injected. After 6 weeks of xenotransplantation, the tumor volume was significantly increased in OS-TIC-transplanted mice compared with recipients transplanted with parental U2OS cells at the same injected cell numbers $\left(2 \times 10^{5}\right.$ cells $)$ (Fig. 3C; $\mathrm{P}<0.05$ ). Furthermore, survival curve analysis indicated that the mean survival rate of OS-TIC-transplanted recipients was significantly lower compared with the parental U2OS cells (Fig. 3D; P<0.01). 
A

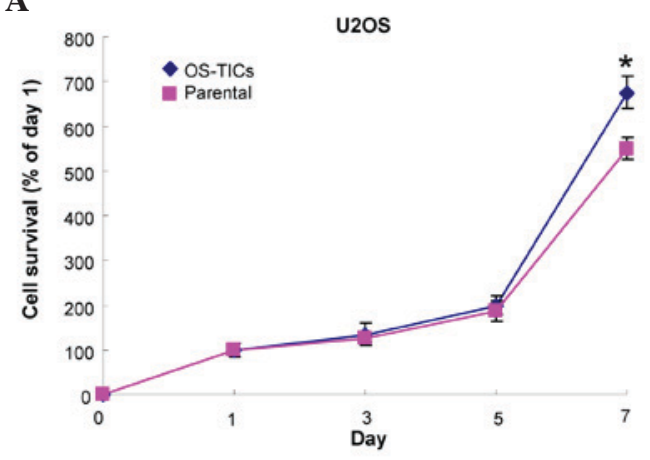

C

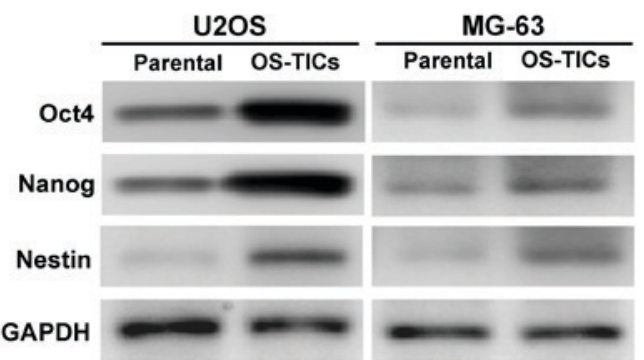

B

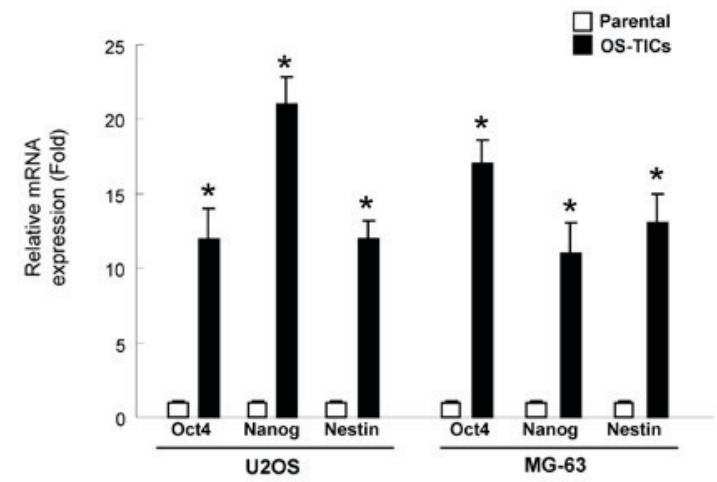

D

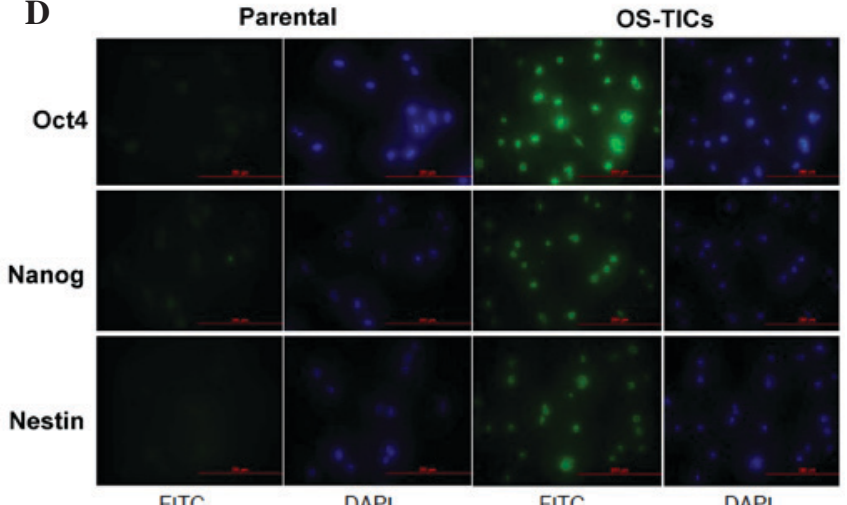

Figure 2. Detection of the expression levels of stemness markers in OS-TICs and parental osteosarcoma cells. (A) Proliferation rate of parental and OS-TICs was determined by 3-(4,5-dimethyl thiazol)-2,5-diphenyltetrazolium bromide assay. (B) Quantitative reverse transcription-polymerase chain reaction analysis revealed the transcription amounts of Nanog, Oct-4 and Nestin in OS-TICs and parental osteosarcoma cells. (C) Total proteins were prepared from parental osteosarcoma or OS-TICs and analyzed by immunoblotting with anti-Oct-4, -Nanog, -Nestin or-GAPDH antibodies as indicated. The amount of GAPDH protein of various crude cell extracts was referred as loading control. (D) By immunofluorescence analysis, parental and enriched OS-TICs from U2OS cells were stained with anti-Oct-4 (top panel), anti-Nestin (middle panel) or anti-Nanog, respectively. ${ }^{*} \mathrm{P}<0.05$. OS-TICs, osteosarcoma-tumor-initiating cells; Oct-4, octamer-binding transcription factor 4; Nanog, Nanog homeobox; FITC, fluorescein isothiocyanate.

A

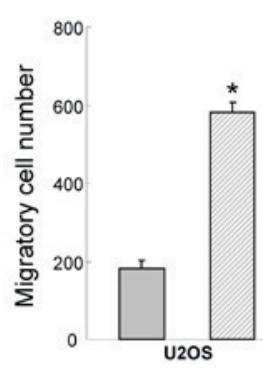

C

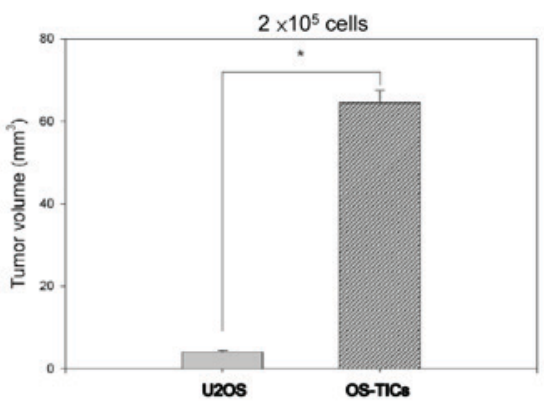

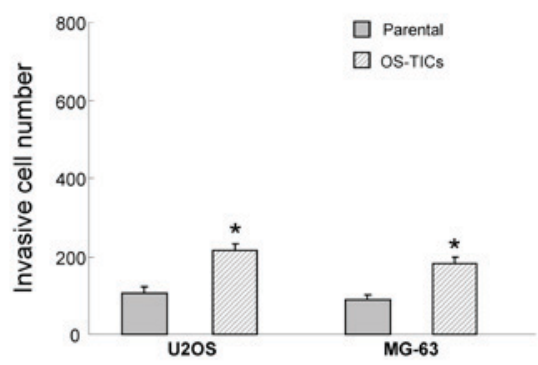

D

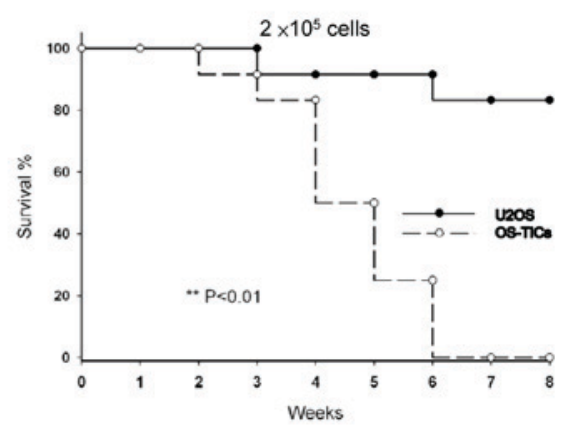

Figure 3. Elevation of the tumorigenicity of OS-TICs in vitro and in vivo. To elucidate the capabilities of migration/invasion of parental osteosarcoma and derived OS-TICs, single-cell suspension of parental or OS-TICs were plated onto (A) Transwell or (B) Matrigel coated Transwell chambers and analyzed $($ ( $\mathrm{P}<0.05)$. (C) Total tumor volume was compared in nude mice injected with the same number $\left(2 \times 10^{5}\right)$ of parental U2OS cells and OS-TICs after 6 weeks of xenotransplantation ( $\mathrm{P}<0.05)$. (D) Survival curves of mice injected with the same number $\left(2 \times 10^{5}\right)$ of parental U2OS cells (solid circle with solid line) and derived OS-TICs (open circle with dashed line) were examined ("P<0.05). OS-TICs, osteosarcoma tumor-initiating cells. 
A

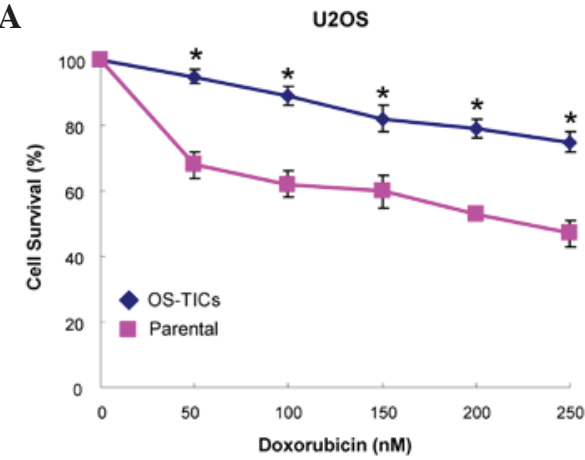

B

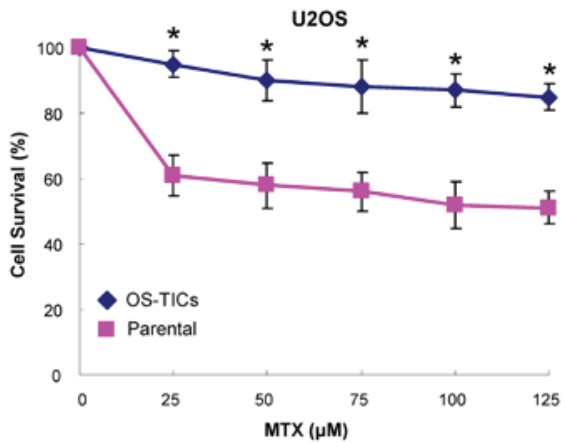

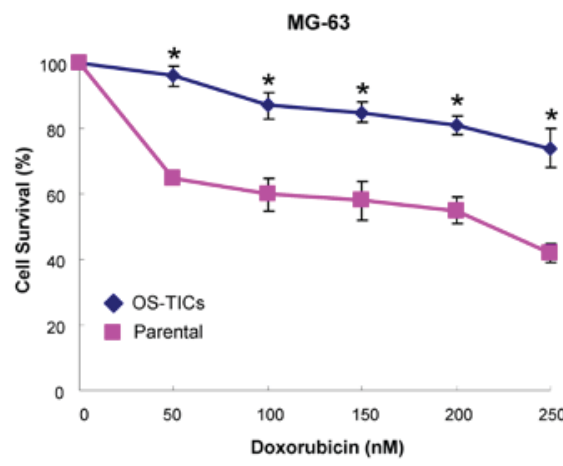

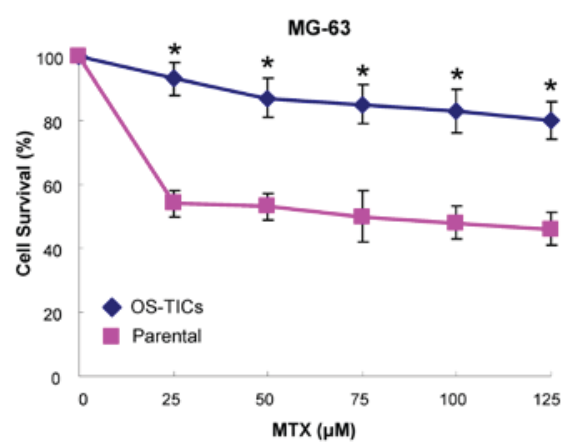

Figure 4. Evaluation of cytotoxic effects of doxorubicin and MTX on OS-TICs and parental osteosarcoma cells. Parental and OS-TICs osteosarcoma cells were subjected to treatment with various concentrations of (A) doxorubicin or (B) MTX. Cell viability was determined by 3-(4,5-dimethyl thiazol)-2,5-diphenyltetrazolium bromide assay ("P<0.05). MXT, methotrexate; OS-TICs, osteosarcoma tumor-initiating cells.

A

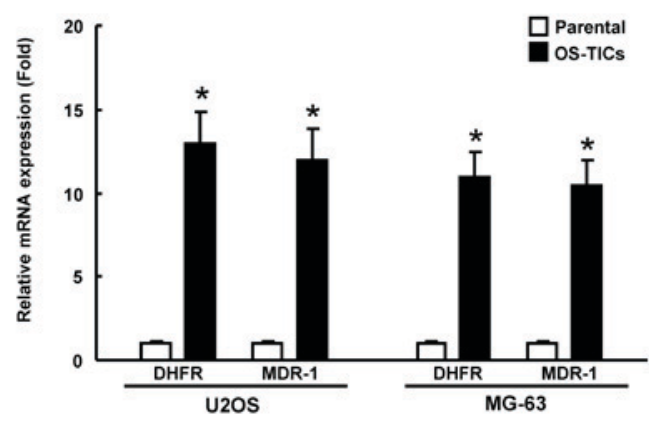

B

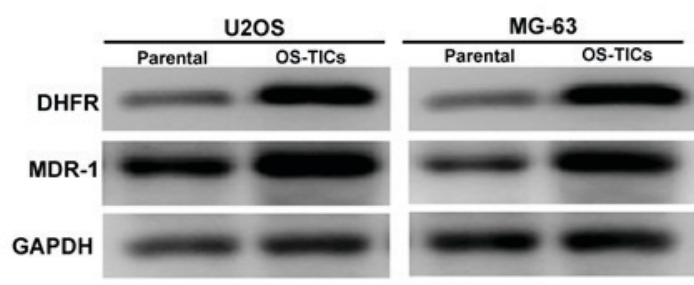

C

Parental

OS-TICS

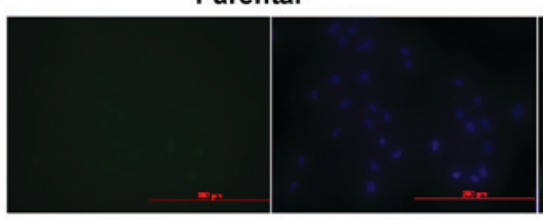

FITC

DAPI

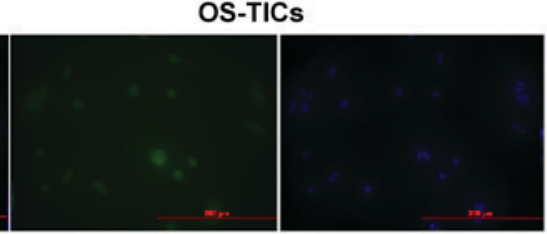

FITC

DAPI

Figure 5. Upregulation of DHFR and MDR1 expression in OS-TICs. (A) Total RNA was purified from parental and OS-TICs, and the elevated expression of DHFR and MDR1 genes in derived OS-TICs was detected by quantitative reverse transcription-polymerase chain reaction analysis. (B) Total proteins were prepared from parental or OS-TICs cells and analyzed by immunoblotting with anti-DHFR, anti-MDR1 or anti-GAPDH antibodies as indicated. The amount of GAPDH protein of different crude cell extracts was referred as loading control. (C) Parental and enriched OS-TICs from U2OS cells were stained with anti-MDR1 to detect the intracellular level of MDR1 proteins by immunofluorescence analysis. " $\mathrm{P}<0.05$. DHFR, dihydrofolate reductase; MDR1, multidrug resistance protein 1; OS-TICs, osteosarcoma tumor-initiating cells; FITC, fluorescein isothiocyanate; DAPI.

Enhanced resistance of OS-TICs to chemotherapy. Resistance to chemotherapy treatment is the major clinical criterion to characterize CSCs (12). Accordingly, an MTT assay was used to monitor the sensitivity of OS-TICs to the treatment of MTX and doxorubicin. Notably, OS-TICs were more chemoresistant to doxorubicin (Fig. 4A) and MTX (Fig. 4B) in dose-dependent concentrations compared with parental U2OS cells $(\mathrm{P}<0.05)$. 
Elevated expression of DHFR and MDR1 genes in OS-TICs. Since chemoresistance to doxorubicin and MTX is enhanced in enriched OS-TICs, the present study sought to investigate the mechanisms underlying this regulation of chemoresistance. DHFR, a target of MTX, was previously shown to regulate the acquisition of chemoresistance to MTX in human osteosarcoma cells $(7,8)$. MDR1, a member of ATP-binding cassette (ABC) transporter superfamily, is critical for regulating the resistance to multiple chemotherapeutic drugs (13). The expression of DHFR and MDR1 mRNA was examined transcriptionally by qRT-PCR. The total RNA of parental U2OS cells or OS-TICs was extracted. The amount of DHFR and MDR1 transcripts of enriched OS-TICs was significantly increased compared with parental U2OS cells (Fig. 5A). In accordance with the results of the qRT-PCR, the western blot analysis also showed that DHFR and MDR1 were also upregulated at the protein level in enriched OS-TICs (Fig. 5B). Furthermore, immunofluorescent staining showed that MDR1 expression was dramatically increased in OS-TICs derived from U2OS cells, compared with those in parental U2OS cells (Fig. 5C).

\section{Discussion}

Osteosarcomas are highly aggressive tumors, which most commonly occur in children and young adults (13). Previous studies have demonstrated the existence of a highly tumorigenic cell subset, commonly known as CSCs, within the tumor bulk (14), and the existence of CSCs within osteosarcoma has also been documented in several studies (15-20). CD117-positive osteosarcoma cells show highly metastatic, tumorigenic capacity and resistance to chemotherapy (17). CD133-positive osteosarcoma cells also possess CSC features, including a high proliferation rate, enhanced abilities for sphere cluster formation, clonogenic efficiency and in vivo tumorigenicity $(18,20)$. In addition, the embryonic stem cell-specific transcriptional factor, sex determining region Y-box 2, also maintains self-renewal and tumorigenic properties in osteosarcoma CSCs (15). In the present study, OS-TICs were found to express ABCG2, a membrane-associated protein that is usually associated with side population phenotype and ATP-dependent exclusion of cellular toxic agents (21). Given that the expression of ABC transporters, including MDR1 and ABCG2, may be important for multidrug resistance to chemotherapeutic agents (22), the expression of ABCG2 can be considered as an additional biomarker for the identification of OS-TICs.

Oct-4 and Nanog were previously suggested as two of the four major factors that render the reprogramming capability of adult cells into germ-line-competent induced pluripotent stem cells $(23,24)$. Nanog blocks differentiation functionally, and the clinical results showed that the elevated expression of Nanog has been associated with retinoblastoma, prostate cancer, embryonal carcinoma, metastatic germ cell tumor and ovarian cancer (25-29). The expression of Oct-4 and Nanog has also been shown in human oral cancer stem-like cells, suggesting that its expression may be implicated in self-renewal and tumorigenesis (10). In the present study, a subpopulation of CSCs from OS-TICs were successfully isolated and enriched using tumor sphere formation assays (Fig. 1A). Notably, the enriched OS-TICs exhibited CSC-like features. For example, the results of immunofluorescent staining and flow cytometry analysis revealed that enriched OS-TICs were stained positive for several stem cell markers, including Oct-4, Nanog, CD133 and CD117, as well as the ABC transporters, MDR1 and ABCG2. Consistent with these findings, another study proposed that aberrant expression of Oct- 4 may contribute to the neoplastic process in cells (3). Overall, these findings indicated that the abnormal expression of Oct-4 or Nanog in stem cells may be critical for regulating tumorigenicity $(30,31)$. The chemoresistant properties of CSCs have been associated with the expression of Oct-4 and Nanog (32-36). In one study, the overexpression of Nanog promoted cisplatin resistance and decreased the proportion of apoptotic cells in esophageal cancer cells (36). Another study found that the ectopic overexpression of Oct-4 and Nanog enhanced the mRNA levels of $\mathrm{ABCB} 1$, resulting in an increased tolerance of lung adenocarcinoma cells to cisplatin treatment (32). Further studies on the regulatory networks between Oct-4 or Nanog and chemoresistance may be required to update the current knowledge for the future development of therapies against osteosarcoma.

Doxorubicin is widely used as a chemotherapeutic agent in the treatment of malignant cancers, including osteosarcoma $(37,38)$. The cytotoxic effects mediated by doxorubicin on malignant cells have been shown to involve: i) DNA base-pair intercalation; ii) the interaction of drug molecules with topoisomerase II to induce the formation of DNA-cleavable complexes; and iii) the interaction of drug molecules with electron transport chain, which may result in the generation of superoxide anion radicals in the cells (39). The mechanisms involved in the chemoresistance of tumor cells to doxorubicin have been shown to include: i) The overexpression of membrane-associated efflux pump, P-glycoprotein, mediating multidrug resistance; ii) the altered expression of topoisomerase II and integrins; and iii) altered glutathione levels (40). P-glycoprotein, a product of the MDR1 gene, is an ATP-dependent drug efflux pump that extrudes drugs from cells and eventually results in the resistance of cells to chemotherapy (41). Numerous studies have also shown that doxorubicin is involved in acquired multidrug resistance mediated by the expression of P-glycoprotein in drug-resistant tumor cells of osteosarcoma $(42,43)$. The results of the present study implied that the enhanced resistance of osteosarcoma CSCs to doxorubicin could be associated with the upregulation of P-glycoprotein.

MTX is another widely-used anticancer drug for chemotherapy against osteosarcoma $(14,44,45)$. Several lines of evidence have demonstrated that DHFR is critical in the regulation of chemoresistance to MTX in human osteosarcoma cells $(7,8)$. However, the causal association of DHFR with chemoresistance to MTX has been extensively investigated; therefore, the present study did not examine additional data addressing the mechanisms by which DHFR may affect the drug responsiveness in human osteosarcoma patients. In agreement with the previous reports, the present study also validated the increased DHFR gene and protein expression in the enriched OS-TICs that were MTX-resistant. These results also indicate that DHFR-mediated MTX resistance in osteosarcoma may be associated with the phenotype of CSCs. Additional studies will be required to clarify the molecular mechanisms responsible for the DHFR-mediated MTX resistance in OS-TICs in more detail. 
The findings of the present study demonstrate that stemness/self-renewal genes, including Oct-4 and Nanog could be important for osteosarcoma-derived stem-like cells. The present study also found that CSCs/TICs were involved in multidrug resistance of osteosarcoma, and that the upregulation of MDR1 and DHFR were responsible for the resistance to anticancer drugs in human OS-TICs. These findings may be beneficial to the development of novel anticancer therapeutic strategies, since these identified genes may represent attractive targets against human osteosarcoma.

\section{Acknowledgements}

The present study was supported by research grants from Chung Shan Medical University Hospital, Taichung, Taiwan, R.O.C. (grant no. CSH-2010-C-025).

\section{References}

1. Siegel R, Naishadham D and Jemal A: Cancer statistics, 2013. CA Cancer J Clin 63: 11-30, 2013.

2. Fan YL, Zheng M, Tang YL and Liang XH: A new perspective of vasculogenic mimicry: EMT and cancer stem cells (Review). Oncol Lett 6: 1174-1180, 2013.

3. Gidekel S, Pizov G, Bergman Y and Pikarsky E: Oct-3/4 is a dose-dependent oncogenic fate determinant. Cancer Cell 4: 361-370, 2003.

4. Nichols J,Zevnik B, Anastassiadis K, Niwa H, Klewe-Nebenius D, Chambers I, Schöler H and Smith A: Formation of pluripotent stem cells in the mammalian embryo depends on the POU transcription factor Oct4. Cell 95: 379-391, 1998.

5. Chambers I, Colby D, Robertson M, Nichols J, Lee S, Tweedie S and Smith A: Functional expression cloning of Nanog, a pluripotency sustaining factor in embryonic stem cells. Cell 113: 643-655, 2003.

6. Costi MP and Ferrari S: Update on antifolate drugs targets. Curr Drug Targets 2: 135-166, 2001.

7. Hattinger CM, Reverter-Branchat G, Remondini D, Castellani GC, Benini S, Pasello M, Manara MC, Scotlandi K, Picci P and Serra M: Genomic imbalances associated with methotrexate resistance in human osteosarcoma cell lines detected by comparative genomic hybridization-based techniques. Eur J Cell Biol 82: 483-493, 2003.

8. Serra M, Reverter-Branchat G, Maurici D, Benini S, Shen JN, Chano T, Hattinger CM, Manara MC, Pasello M, Scotlandi K and Picci P: Analysis of dihydrofolate reductase and reduced folate carrier gene status in relation to methotrexate resistance in osteosarcoma cells. Ann Oncol 15: 151-160, 2004.

9. Jordan CT, Guzman ML and Noble M: Cancer stem cells. N Engl J Med 355: 1253-1261, 2006.

10. Chiou SH, Yu CC, Huang CY, Lin SC, Liu CJ, Tsai TH, Chou SH, Chien CS, Ku HH and Lo JF: Positive correlations of Oct-4 and Nanog in oral cancer stem-like cells and high-grade oral squamous cell carcinoma. Clin Cancer Res 14: 4085-4095, 2008.

11. Livak KJ and Schmittgen TD: Analysis of relative gene expression data using real-time quantitative PCR and the 2(-Delta Delta C(T)) Method. Methods 25: 402-408, 2001.

12. Wang JC: Evaluating therapeutic efficacy against cancer stem cells: New challenges posed by a new paradigm. Cell Stem Cell 1: 497-501, 2007.

13. Dean M: ABC transporters, drug resistance, and cancer stem cells. J Mammary Gland Biol Neoplasia 14: 3-9, 2009.

14. Marina N, Gebhardt M, Teot L and Gorlick R: Biology and therapeutic advances for pediatric osteosarcoma. Oncologist 9: 422-441, 2004

15. Basu-Roy U, Seo E, Ramanathapuram L, Rapp TB, Perry JA, Orkin SH, Mansukhani A and Basilico C: Sox2 maintains self renewal of tumor-initiating cells in osteosarcomas. Oncogene 31: 2270-2282, 2012.

16. Di Fiore R, Santulli A, Ferrante RD, Giuliano M, De Blasio A, Messina C, Pirozzi G, Tirino V, Tesoriere G and Vento R: Identification and expansion of human osteosarcoma-cancer-stem cells by long-term 3-aminobenzamide treatment. J Cell Physiol 219: 301-313, 2009.
17. Adhikari AS, Agarwal N, Wood BM, Porretta C, Ruiz B, Pochampally RR and Iwakuma T: CD117 and Stro-1 identify osteosarcoma tumor-initiating cells associated with metastasis and drug resistance. Cancer Res 70: 4602-4612, 2010.

18. Tirino V, Desiderio V, d'Aquino R, De Francesco F, Pirozzi G, Graziano A, Galderisi U, Cavaliere C, De Rosa A, Papaccio G and Giordano A: Detection and characterization of CD133+ cancer stem cells in human solid tumours. PLoS One 3: e3469, 2008.

19. Tirino V, Desiderio V, Paino F, De Rosa A, Papaccio F, Fazioli F, Pirozzi G and Papaccio G: Human primary bone sarcomas contain CD133+ cancer stem cells displaying high tumorigenicity in vivo. FASEB J 25: 2022-2030, 2011.

20. Saini V, Hose CD, Monks A, Nagashima K, Han B, Newton DL, Millione A, Shah J, Hollingshead MG, Hite KM, et al: Identification of $\mathrm{CBX} 3$ and $\mathrm{ABCA} 5$ as putative biomarkers for tumor stem cells in osteosarcoma. PLoS One 7: e41401, 2012.

21. Pascal LE, Oudes AJ, Petersen TW, Goo YA, Walashek LS, True LD and Liu AY: Molecular and cellular characterization of ABCG2 in the prostate. BMC Urol 7: 6, 2007.

22. Bunting KD: ABC transporters as phenotypic markers and functional regulators of stem cells. Stem Cells 20: 11-20, 2002.

23. Park IH, Zhao R, West JA, Yabuuchi A, Huo H, Ince TA, Lerou PH, Lensch MW and Daley GQ: Reprogramming of human somatic cells to pluripotency with defined factors. Nature 451: 141-146, 2008.

24. Okita K, Ichisaka T and Yamanaka S: Generation of germline-competent induced pluripotent stem cells. Nature 448: 313-317, 2007.

25. Seigel GM, Hackam AS, Ganguly A, Mandell LM and Gonzalez-Fernandez F: Human embryonic and neuronal stem cell markers in retinoblastoma. Mol Vis 13: 823-832, 2007.

26. Santagata S, Ligon KL and Hornick JL: Embryonic stem cell transcription factor signatures in the diagnosis of primary and metastatic germ cell tumors. Am J Surg Pathol 31: 836-845, 2007.

27. Ma S, Chan KW, Hu L, Lee TK, Wo JY, Ng IO, Zheng BJ and Guan XY: Identification and characterization of tumorigenic liver cancer stem/progenitor cells. Gastroenterology 132: 2542-2556, 2007.

28. Freberg CT, Dahl JA, Timoskainen S and Collas P: Epigenetic reprogramming of OCT4 and NANOG regulatory regions by embryonal carcinoma cell extract. Mol Biol Cell 18: 1543-1553, 2007.

29. Hoei-Hansen CE, Kraggerud SM, Abeler VM, Kaern J, Rajpert-De Meyts E and Lothe RA: Ovarian dysgerminomas are characterised by frequent KIT mutations and abundant expression of pluripotency markers. Mol Cancer 6: 12, 2007.

30. Trosko JE: From adult stem cells to cancer stem cells: Oct-4 Gene, cell-cell communication, and hormones during tumor promotion. Ann N Y Acad Sci 1089: 36-58, 2006.

31. Pan G and Thomson JA: Nanog and transcriptional networks in embryonic stem cell pluripotency. Cell Res 17: 42-49, 2007.

32. Chiou SH, Wang ML, Chou YT, Chen CJ, Hong CF, Hsieh WJ, Chang HT, Chen YS, Lin TW, Hsu HS and Wu CW: Coexpression of Oct4 and Nanog enhances malignancy in lung adenocarcinoma by inducing cancer stem cell-like properties and epithelial-mesenchymal transdifferentiation. Cancer Res 70: 10433-10444, 2010.

33. Zhang J, Espinoza LA, Kinders RJ, Lawrence SM, Pfister TD, Zhou M, Veenstra TD, Thorgeirsson SS and Jessup JM: NANOG modulates stemness in human colorectal cancer. Oncogene 32: 4397-4405, 2013

34. Jeter CR, Liu B, Liu X, Chen X, Liu C, Calhoun-Davis T, Repass J, Zaehres H, Shen JJ and Tang DG: NANOG promotes cancer stem cell characteristics and prostate cancer resistance to androgen deprivation. Oncogene 30: 3833-3845, 2011.

35. Ibrahim EE, Babaei-Jadidi R, Saadeddin A, Spencer-Dene B, Hossaini S, Abuzinadah M, Li N, Fadhil W, Ilyas M, Bonnet D and Nateri AS: Embryonic NANOG activity defines colorectal cancer stem cells and modulates through AP1- and TCF-dependent mechanisms. Stem Cells 30: 2076-2087, 2012.

36. Yang L, Zhang X, Zhang M, Zhang J, Sheng Y, Sun X, Chen Q and Wang LX: Increased Nanog expression promotes tumor development and Cisplatin resistance in human esophageal cancer cells. Cell Physiol Biochem 30: 943-952, 2012.

37. Serra M, Scotlandi K, Manara MC, Maurici D, Lollini PL, De Giovanni C, Toffoli G and Baldini N: Establishment and characterization of multidrug-resistant human osteosarcoma cell lines. Anticancer Res 13: 323-329, 1993. 
38. Scotlandi K, Serra M, Manara MC, Lollini PL, Maurici D, Del Bufalo D and Baldini N: Pre-treatment of human osteosarcoma cells with $\mathrm{N}$-methylformamide enhances P-glycoprotein expression and resistance to doxorubicin. Int J Cancer 58: 95-101, 1994.

39. Taylor CW, Dalton WS, Parrish PR, Gleason MC, Bellamy WT, Thompson FH, Roe DJ and Trent JM: Different mechanisms of decreased drug accumulation in doxorubicin and mitoxantrone resistant variants of the MCF7 human breast cancer cell line. Br J Cancer 63: 923-929, 1991.

40. Rajkumar T and Yamuna M: Multiple pathways are involved in drug resistance to doxorubicin in an osteosarcoma cell line. Anticancer Drugs 19: 257-265, 2008.

41. Chen CJ, Chin JE, Ueda K, Clark DP, Pastan I, Gottesman MM and Roninson IB: Internal duplication and homology with bacterial transport proteins in the mdrl (P-glycoprotein) gene from multidrug-resistant human cells. Cell 47: 381-389, 1986.
42. Baldini N, Scotlandi K, Barbanti-Bròdano G, Manara MC, Maurici D, Bacci G, Bertoni F, Picci P, Sottili S, Campanacci M, et al: Expression of P-glycoprotein in high-grade osteosarcomas in relation to clinical outcome. N Engl J Med 333: 1380-1385, 1995

43. Baldini N, Scotlandi K, Serra M, Picci P, Bacci G, Sottili S and Campanacci M: P-glycoprotein expression in osteosarcoma: A basis for risk-adapted adjuvant chemotherapy. J Orthop Res 17: 629-632, 1999.

44. Ferrari S and Palmerini E: Adjuvant and neoadjuvant combination chemotherapy for osteogenic sarcoma. Curr Opin Oncol 19: 341-346, 2007.

45. Chou AJ and Gorlick R: Chemotherapy resistance in osteosarcoma: Current challenges and future directions. Expert Rev Anticancer Ther 6: 1075-1085, 2006. 